\title{
Meiosis 2007 - Where have we got to and where are we going?
}

\author{
James M. A. Turner* \\ MRC Biomedical NMR Centre, National Institute for Medical Research, The Ridgeway, Mill Hill, London, UK; \\ E-mail: jturner@nimr.mrc.ac.uk \\ *Correspondence
}

At first glance, the fundamental principle of meiosis is a straightforward mathematical one: a diploid cell undergoes a single round of DNA replication followed by two rounds of cell division, generating haploid products, which in most organisms subsequently unite to create a new organism with the diploid chromosome number. The reality, however, is that meiosis is far more complicated. First of all, cells must make an active decision to opt out of the more common form of cell division mitosis. Following commitment to meiosis and DNA replication, the chromosomes must then engage in physical pairing with their homologous partners - a process known as synapsis - accompanied by telomere clustering and the reciprocal and nonreciprocal exchange of genetic material by homologous recombination. Reciprocal exchanges, known as chiasmata, must be formed in the correct numbers and be properly spaced along the chromosome arms in order to ensure that chromosome segregation then occurs normally during the first meiotic division. All of these different processes have to be carefully co-ordinated, and if one or more goes awry, the cell is eliminated in order to prevent the generation of aneuploid daughter cells.

In this special edition of Chromosome Research, we have compiled a series of reviews covering the successive hurdles that meiotic cells must pass in order to generate haploid daughters. In the first review, Harigaya and Yamamoto discuss intriguing new findings on the mitosis-meiosis decision in Schizosaccharomyces pombe (fission yeast). In this organism meiosis is actively suppressed during vegetative growth by the selective elimination of transcripts encoding meiotic genes. Remarkably, these transcripts harbour molecular tags - the so-called DSRs (determinant of selective removal) that render them susceptible to removal by the RNAbinding protein Mmil. Inactivation of the DSRMmil system is a key step in the mitosis - meiosis switch and is mediated by Mei2 (Harigaya et al. 2006). In conditions where meiosis is triggered, e.g following nutrient starvation, expression of Mei2 is stimulated, and the resulting Mei2 protein sequesters Mmi1 at a discrete 'dot' in the meiotic nucleus where it can no longer orchestrate the elimination of meiotic transcripts. This post-transcriptional mode of silencing meiotic genes in non-meiotic cells is advantageous presumably because it can be more rapidly reversed in conditions where meiosis needs to be initiated.

Having embarked on meiosis, meiotic cells must then begin the task of homologous recombination (HR). The early stages of recombination are critically dependent upon the combined actions of three proteins; Mre11, Rad50 and Xrs2 (the Saccharomyces cerevisiae - or budding yeast - orthologue of mammalian NBS1), collectively known as the Mre11 complex. In her review, Valerie Borde explains that this complex performs at least three functions in meiosis. Firstly, it forms a complex on DNA that in many, but not all, organisms is required for the earliest step in the recombination process - the formation of DNA double strand breaks (DSBs) by the topoisomerase Spo11. Once breaks are formed, Spo11 becomes covalently attached to the break site 
and must be removed before break resection and strand invasion can take place. Here, the MRE11 complex is again crucial. The nuclease activity of Mre11, whose function in meiotic recombination remained elusive for many years, evicts Spo11 together with a short 15-30bp DNA oligonucleotide from the break site, thus allowing resection to proceed (Neale et al. 2005). Finally, all DSBs must be resolved prior to the initiation of the meiotic divisions, and therefore breaks must be sensed and transduced into a signal that causes cell cycle arrest. This is achieved through Mre11 complex-dependent activation of the PI3-like checkpoint kinase Atm.

Atm, together with Atr, are members of the PI3like kinase family, whose budding yeast orthologues are Tel1 and Mec1, respectively. These are chromosome-associated signal transduction kinases that phosphorylate a bewildering array of recombination and repair proteins following the induction of DNA DSBs (Matsuoka et al. 2007), and this phosphorylation serves principally to facilitate protein-proteins interactions. Our traditional view of ATM and ATR was that they function solely as checkpoint proteins, but this view is challenged in a review by Carballo and Cha. They stress that Mec1 is required for various chromosomal processes during both mitosis and meiosis even in the unchallenged state. For instance, Mec1 is required for DNA replication through regions of the genome termed Replication Slow Zones - this slowing of replication may be important for the installation of specific chromosomeassociated proteins such as nucleosomes and cohesins. Furthermore, mutations in Mec1 confer specific meiotic phenotypes - programmed DSBs tend to be repaired using the sister chromatid rather than the homologous chromosome, and the normal non-random distribution of crossovers is disturbed. Based on these observations, the authors propose that ATM/ATR function principally to co-ordinate and promote fundamental chromosome events, rather than as checkpoint proteins in the traditional sense.

Spo11-catalysed DNA DSBs are formed in large numbers during meiosis, but only a select number proceed to form the crossovers (COs) that are so vital in ensuring normal chromosome segregation. For instance, in male mice, between 200 and 400 DSBs are formed during leptotene, but only around 23 form COs. What mechanisms determine which DSBs are destined to form crossovers and how are the remaining DSBs resolved? Strong evidence now exists showing that most non-crossover (NCO) destined breaks are resolved as gene conversion events between homologues. NCOs and COs are now known to result from distinct repair pathways (Allers \& Lichten, 2001). An important player in the CO pathway is the ZMM (Zip1 through Zip4, Msh4/ Msh5, Mer3) protein family, and the functions of each of these members are discussed by Lynn, Soucek and Borner. In yeast, ZMM proteins form foci whose numbers approximate the number of COs formed (around 60-70) and mutations in any of the seven components abolish the formation of most COs without affecting NCOs. However, in other organisms, ZMM proteins may also be required for $\mathrm{NCO}$ formation. As discussed by Baudat and de Massy, the numbers of Msh4 foci in mouse meiocytes far exceeds the number of $\mathrm{CO}$ events, and mutations in Msh4 cause defects in both COs and NCOs. These findings suggest that the ZMM proteins might function before the separation of $\mathrm{NCO}$ and $\mathrm{CO}$ pathways in mice, and highlight the fact that fundamental properties of meiosis differ from organism to organism. Another example of such variation relates to crossover interference - the property by which the occurrence of a $\mathrm{CO}$ at a defined point on a homologous pair reduces the likelihood of another $\mathrm{CO}$ occurring within close proximity. In budding yeast, COs but not NCOs exhibit interference, but in mice interference is observed for NCOs, albeit at a lower level than COs (de Boer et al. 2006).

A defining feature in meiosis is the formation of the synaptonemal complex (SC), a proteinaceous 'zip' that acts to align maternal and paternal homologues (de Boer \& Heyting 2006). Originally, the SC was regarded as a tripartite structure: two lateral elements (LE) are formed between the sisters of each homologue, and these are joined by transverse filaments (TF) which lie perpendicular to the lateral elements. Although a third, densely staining structure has been observed along the centre of the $\mathrm{SC}$ - the so-called central element (CE) - this was thought to reflect overlap between opposed TFs. New findings in the mouse now show that the CE actually represents a distinct structure with its own protein complement, implying that the SC is in fact quadripartite (Costa et al. 2005; Hamer et al. 2006). The role of the $\mathrm{CE}$, together with that of the $\mathrm{LE}$ and $\mathrm{TF}$, are discussed in a review by Costa and Cooke. They explain how different components of the SC function in meiotic synapsis, $\mathrm{CO}$ formation and normal 
chromatin architecture. They also highlight how targeted mutations in SC components, e.g. the LE proteins SYCP2 and SYCP3 give sexually dimorphic effects, with males being sterile and females subfertile. In Sycp3 mutant females, CO formation is disturbed and as a consequence chromosomes missegregate, giving rise to oocytes and thereafter embryos with the wrong chromosome number. This has provided a powerful tool for studying the effects of aneuploidy on mouse embryonic development (Lightfoot et al. 2006).

In mice, yeast and Arabidopsis thaliana, meiotic DNA DSB formation is essential for normal meiotic synapsis (Keeney 2001). DSBs are thought to drive synapsis through the presence of resected, single stranded tails, which perform a Rad51-mediated homology search and thereby bring the broken DNA molecule into close physical proximity with its uncut homologous partner. However, this is not the case Caenorhabditis elegans (flatworm) - chromosomes synapse normally in a Spo11 mutant, although the lack of DSB formation and hence CO formation nevertheless leads to later chromosome mis-segregation (Dernburg et al. 1998). If synapsis is DSB-independent in C. elegans, how else could it be mediated? This and other intriguing aspects of meiosis in c.elegans are discussed by Garcia-Muse and Boulton. One of the notable recent developments in research in this organism is the discovery of a set of zinc-finger proteins HIM-8, ZIM-1, ZIM-2 and ZIM-3, which mediate pairing of specific chromosomes through interactions with specialised chromosome-specific pairing centres (PCs; Phillips \& Dernburg 2006). Correct targeting of the ZIM proteins to PCs has been unexpectedly linked to the checkpoint kinase chk2, which could modulate ZIM function directly through phosphorylation.

A characteristic and highly conserved feature of early meiosis is the formation of the bouquet (Scherthan 2001). This structure results from clustering of chromosome ends - or telomeres - to a restricted site on the nuclear membrane that sits next to the centrosome in mammals and spindle pole body in fission yeast. Because bouquet formation brings homologous chromosomes into close proximity, it has been suggested that it serves an important role in homologous interactions. As discussed in a review on telomeres by Siderakis and Tarsounas, this is supported by the finding that mutations in telomereassociated proteins such as Ndj1p in budding yeast and Taz1 in fission yeast disrupt bouquet formation and HR (Trelles-Sticken et al. 2000; Cooper et al. 1998). The authors also cover other interesting aspects of telomere biology, including how telomeric DNA avoids being recognised as DNA damage and thus triggering cell cycle arrest. The answer may lie in the fact that telomeres form a large duplex loop, created by invasion of the $3^{\prime}$ telomeric overhang into the duplex DNA of the same telomere (Griffith et al. 1999). This invasion is highly reminiscent of that which takes place during HR and the authors report that a component of the RAD51 paralogue family, RAD51D, as well as other HR proteins such as FANCD2 localise to telomeres during meiosis.

Homologous synapsis and recombination are accompanied by dramatic changes in chromosome conformation, and no more striking example of this exists than Meiotic Sex Chromosome Inactivation (MSCI; Turner 2007). MSCI refers to the transcriptional silencing of the $\mathrm{X}$ chromosome that takes place in the male in species with heteromorphic sex chromosomes, i.e. males $\mathrm{XY}$ or $\mathrm{XO}$, females $\mathrm{XX}$. MSCI represents a remarkable example of how meiotic chromosome pairing influences gene expression. In male flatworms (XO), the single X chromosome is enriched in histone marks associated with transcriptional silencing. This is because it has no homologous partner, because when autosomes fail to synapse, they acquire the same repressive marks (Bean et al. 2004). A comparable situation exists in mice - the unsynapsed $\mathrm{X}$ and $\mathrm{Y}$ chromosome are subject to MSCI during pachytene in the male, but when other chromosomes fail to synapse, they are also silenced, both in male and female meiosis (Baarends et al. 2005; Turner et al. 2005). These observations have revealed the existence of a highly conserved mechanism that silences any chromosome that fails to find its homologous partner - called Meiotic Silencing of Unsynapsed Chromatin (MSUC). The molecular genetics, evolution and functions of MSUC are only beginning to be elucidated and are discussed by Kelly and Aramayo.

The existence of MSCI is one possible explanation for why genes functioning in male germ cell development are under-represented on the flatworm $\mathrm{X}$ chromosome (Kelly et al. 2002). Surprising, a study by Wang et al. (2001) has found that spermatogenesis genes are actually enriched on the mouse $\mathrm{X}$ chromosome, despite the fact that MSCI also operates in this organism. This paradox is 
discussed in a review by Wang and Pan. They explain that $\mathrm{X}$-linked spermatogenesis genes are expressed prior to the onset of MSCI - in spermatogonia. These genes were identified as part of a screen to identify spermatogonia-specific transcripts, and targeted mutations of many of the autosomal spermatogonial genes have since been shown to result in meiotic phenotypes. Wang and Pan speculate that similar phenotypes may result from mutations of the $\mathrm{X}$-linked spermatogonial-specific genes as well. If so, this would represent one of the first examples of an $\mathrm{X}$-linked gene required for mouse meiosis.

Staying on the theme of epigenetics, Holmes and Cohen then discuss the roles of small RNAs and RNAi pathways in meiosis, focusing on recent advances on the PIWI proteins and their associated small RNAs (piRNAs). The PIWI proteins are members of the Argonaute family, which function in the RNAi pathway by binding Dicer-derived small RNAs and targeting homologous mRNAs for degradation. Several members of the Piwi family are expressed in the germ line, and these associate with a huge number of piRNAs, around one million per meiotic cell (reviwed in Seto et al. 2007). Mutations of Piwi members result in male sterility and inappropriate transcription of transposable elements, implying a role for piRNAs in transposon control, as found previously for Drosophila Piwi orthologues. However, since most piRNAs are depleted of repeat sequences, they may serve additional functions in germ cell development, perhaps in one or more steps in meiosis. In this regard, it is interesting that mutations of two of the Piwi members, Mili and Miwi2, result in meiotic arrest around the time that recombination and synapsis are taking place (Kuramochi-Miyagawa et al. 2004; Carmell et al. 2007). Holmes and Cohen speculate that Piwi proteins may be involved in centromere stabilisation during meiosis, defects of which would predictably disrupt recombination and synapsis, or that they may function in MSCI.

Assuming that synapsis and recombination have gone according to plan, homologous chromosomes should enter the final stage of meiosis - the meiotic divisions - as bivalents connected by chiasmata. In most organisms - with exceptions including male Drosophila - chiasmata are absolutely critical for normal chromosome segregation to occur. In females, in which recombination is highly error prone, errors in chromosome segregation are com- mon and this is reflected in the extremely high incidence of aneuploidy in humans - affecting 10-30\% of clinically recognised pregnancies (Hassold $\&$ Hunt 2001). Chiasmata serve to counteract the pulling forces exerted by spindle microtubules and this ensures that bivalents align themselves properly on the spindle equator prior to their separation. The classical view of spindle assembly in animals is that they originate from centrosomes situated at the poles of the nucleus but, as discussed by Doubilet and McKim, in some organisms including flies and mammals, microtubules are formed in the absence of centrosomes. Instead, the chromosomes themselves have an active role in the formation of microtubules. The authors discuss key steps in the formation of the acentrosomal spindle, and suggest that this unusual form of chromosome segregation might be linked to the high incidence of aneuploidy in mammals.

These diverse reviews have been selected to illustrate the complexity of meiotic chromosome dynamics and to highlight some of the more important questions that those of us studying this fascinating biological puzzle face. As guest editor, I hope you find them stimulating. I would to extend my thanks to all the authors that contributed to this special issue, as well as to Herbert Macgregor, Editor-in-Chief of Chromosome Research, for inviting me to plan and guest edit this project and for helping me with its preparation.

\section{References}

Allers T, Lichten M (2001) Differential timing and control of noncrossover and crossover recombination during meiosis. Cell 106: $47-57$.

Baarends WM, Wassenaar E, van der Laan R (2005) Silencing of unpaired chromatin and histone $\mathrm{H} 2 \mathrm{~A}$ ubiquitination in mammalian meiosis. Mol Cell Biol 25: 1041-1053.

Bean CJ, Schaner CE, Kelly WG (2004) Meiotic pairing and imprinted X chromatin assembly in Caenorhabditis elegans. Nat Genet 36: 100-105.

Carmell MA, Girard A, van de Kant HJ et al. (2007) MIWI2 is essential for spermatogenesis and repression of transposons in the mouse male germline. Dev Cell 12: 503-514.

Cooper JP, Watanabe Y, Nurse P (1998) Fission yeast Taz1 protein is required for meiotic telomere clustering and recombination. Nature 392: 828-831.

Costa Y, Speed R, Ollinger R (2005) Two novel proteins recruited by synaptonemal complex protein 1 (SYCP1) are at the centre of meiosis. J Cell Sci 118: 2755-2762. 
de Boer E, Heyting C (2006) The diverse roles of transverse filaments of synaptonemal complexes in meiosis. Chromosoma 115: 220-234.

de Boer E, Stam P, Dietrich AJ et al. (2006) Two levels of interference in mouse meiotic recombination. Proc Natl Acad Sci 103: 9607-9612.

Dernburg AF, McDonald K, Moulder G et al. (1998) Meiotic recombination in $C$. elegans initiates by a conserved mechanism and is dispensable for homologous chromosome synapsis. Cell 94: 387-398.

Griffith JD, Comeau L, Rosenfield S, Stansel RM, Bianchi A, Moss H, de Lange T (1999) Mammalian telomeres end in a large duplex loop. Cell 97: 503-514.

Hamer G, Gell K, Kouznetsova A et al. (2006) Characterization of a novel meiosis-specific protein within the central element of the synaptonemal complex. J Cell Sci 119: 4025-4032.

Harigaya Y, Tanaka, H, Yamanaka S et al. (2006) Selective elimination of messenger RNA prevents an incidence of untimely meiosis. Nature 442: 45-50.

Hassold T, Hunt P (2001) To err (meiotically) is human: the genesis of human aneuploidy. Nat Rev Genet 2: 280-291.

Keeney S (2001) Mechanism and control of meiotic recombination initiation. Curr Top Dev Biol 52: 1-53.

Kelly WG, Schaner CE, Dernburg AF et al. (2002) X-chromosome silencing in the germline of C. elegans. Development 129: 479-492.

Kuramochi-Miyagawa S, Kimura T, Ijiri TW et al. (2004) Mili, a mammalian member of piwi family gene, is essential for spermatogenesis. Development 131: 839-849.
Lightfoot DA, Kouznetsova A, Mahdy E et al. (2006) The fate of mosaic aneuploid embryos during mouse development. Dev Biol 289: 384-394.

Matsuoka S, Ballif BA, Smogorzewska A et al. (2007) ATM and ATR substrate analysis reveals extensive protein networks responsive to DNA damage. Science 316: 1160-1166.

Neale MJ, Pan J, Keeney S (2005) Endonucleolytic processing of covalent protein-linked DNA double-strand breaks. Nature 436: 1053-1057.

Phillips CM, Dernburg AF (2006) A family of zinc-finger proteins is required for chromosome-specific pairing and synapsis during meiosis in C. elegans. Dev Cell 11: 817-829.

Scherthan H (2001) A bouquet makes ends meet. Nat Rev Mol Cell Biol 2: 621-626.

Seto AG, Kingston RE, Lau NC et al. (2007) The coming of age for piwi proteins. Mol Cell 26: 603-609.

Trelles-Sticken E, Dresser ME, Scherthan H (2000) Meiotic telomere protein Ndj1p is required for meiosis-specific telomere distribution, bouquet formation and efficient homologue pairing. J Cell Biol 151: 95-106

Turner JM (2007) Meiotic sex chromosome inactivation. Development 134: 1823-1831.

Turner JM, Mahadevaiah, SK, Fernandez-Capetillo O et al. (2005) Silencing of unsynapsed meiotic chromosomes in the mouse. Nat Genet 37: 41-47.

Wang PJ, McCarrey JR, Yang F, Page DC (2001) An abundance of $\mathrm{X}$-linked genes expressed in spermatogonia. Nat Genet 27: $422-426$. 